\title{
Resolved optical-infrared SEDs of galaxies: universal relations and their break-down on local scales
}

\author{
Stefano Zibetti ${ }^{1}$ and Brent Groves ${ }^{2}$ \\ ${ }^{1}$ Dark Cosmology Centre, Niels Bohr Institute, University of Copenhagen, Juliane Maries Vej \\ 30, DK-2100 Copenhagen, Denmark \\ ${ }^{2}$ Max-Planck-Institut für Astronomie, Königstuhl 17, D-69117 Heidelberg, Germany \\ email: zibetti@dark-cosmology.dk brent@mpia.de
}

\begin{abstract}
A large body of evidence has demonstrated that the global rest-frame optical and IR colours of galaxies correlate well with each other, which can be readily interpreted as a sign of typically smooth star formation histories. However the processes that lead to the observed correlations are contrary: the stellar light that contributes to the optical is readily absorbed by dust which emits in the IR. Thus on small scales we expect these correlations to break down. In this contribution we present our recent results (Zibetti \& Groves 2011) from a pixel-by-pixel multi-wavelength $(u$-band to $8 \mu \mathrm{m})$ analysis of seven nearby galaxies ranging from early- to latetypes. We show that such a break-down occurs already on scales on few $100 \mathrm{pc}$, as a result of the different physical conditions in spatially distinct regions inside the galaxy, as we demonstrate by means of a Principal Component Analysis. Despite the lack of internal correlation between optical and IR within individual galaxies, when the pixels of all galaxies are compared the well known optical-IR colour correlations return, demonstrating that the variance observed within galaxies is limited around a mean which follows the well-known trends. We also examine the extremely strong correlations between the mid IR (Spitzer-IRAC)-NIR colours which extend continuously across all galaxies. These correlations arise from the differing contribution of stellar light and dust to the IRAC bands, enabling us to determine pure stellar colours for these bands, but still demonstrating the need for dust (or stellar) corrections in these bands when being used as stellar (dust) tracers.
\end{abstract}

Keywords. galaxies: general - galaxies: individual: NGC4254 galaxies: photometry galaxies: stellar content infrared: ISM.

\section{Introduction}

The optical to mid IR SED of a galaxy $(350 \mathrm{~nm}$ to $\sim 10 \mu \mathrm{m}$ for the scope of this paper) is characterised by the presence of two distinct regimes, short-ward and long-ward of $\sim 1-2 \mu \mathrm{m}$, respectively. At optical wavelengths we observe the radiation of stars, possibly extincted and reddened by dust. Moving into the mid IR we see a combination of stellar radiation and dust glowing as it re-radiates the energy that has been absorbed from stars, either via thermal mechanisms or molecular transitions (e.g. the PAH bands); dust emission becomes more and more dominant as one moves to longer wavelengths. Nowadays it is an obvious and well established fact that the integrated optical colours of galaxies are well correlated to each other (e.g. Blanton et al. 2003 for an illustration of it with the large statistical sample of the SDSS): this reflects the simple fact that the shape of the SED at these wavelengths is mainly dictated by the mean stellar population age (to first order) and metallicity and modulated by dust extinction. Mid IR colours are also correlated with each other as they reflect, to first order, the balance between 
dust and emission from (old) stars. What is much less obvious is the correlation between integrated optical and mid IR colours, in the sense that optically bluer galaxies are normally relatively brighter in the mid IR and, vice versa, red galaxies (unless heavily dust obscured) are dim in mid IR (e.g. Hogg et al. 2005). This correlation indicates that on galaxy scale the hot dust and PAH emission which trace the most recent star formation (on $\sim 10^{7}$ yr timescale, Calzetti et al. 2007) is associated with the diffuse presence of a young stellar component whose age spans up to $1 \mathrm{Gyr}$, as reflected in the optical colours. Such a correlation between integrated optical and mid IR colours is basically an indication of the typical smoothness of the star formation history (SFH) of galaxies.

There is no simple physical reason why such correlations may apply also within galaxies on sub-kpc scales. In fact, on a purely physical ground the interplay between stars and dust can be very complex; in particular, the hot optical-UV radiation needed to power the (hot) dust emission has to be promptly absorbed by the dust itself, with the side effect of reddening the optical spectrum. In fact only a fine tuned balance between the local density of young stars and dust can result in tight optical-mid IR colour correlations.

In order to explore this issue we have performed pixel-by-pixel SED analysis of a small sample of seven nearby $(D \lesssim 20 \mathrm{Mpc})$ galaxies with available imaging in the optical from the SDSS (York et al. 2000, $u, g, r, i, z$ ), in the near IR $H$-band (either from GOLDMine, Gavazzi et al. 2003, or UKIDSS Lawrence et al. 2007) and mid IR in the Spitzer-IRAC bands (from SINGS, Kennicutt et al. 2003), plus narrow-band $\mathrm{H} \alpha$. These galaxies span the full range of mass and morphologies of normal non-dwarf galaxies. We refer the reader to Zibetti \& Groves (2011) for a fully detailed description of the analysis and results; in this contribution we briefly summarise them and highlight the most important points.

\section{Main Results}

The images of each galaxy in the ten broad bands (plus $\mathrm{H} \alpha$ ) are sampled with pixels of approximately $200 \mathrm{pc}$ on a side. For each of these pixels the local SED is constructed by normalising the monochromatic flux $\nu f_{\nu}$ in each band to the flux in $H$ band. This not only allows to study all SEDs on a similar scale but also is optimal in separating the two wavelength regimes dominated by stellar and dust emission respectively. For each galaxy individually we study the distribution of pixels in all possible combinations of "colour-colour" plots (where by "colour" we actually mean $H$-band normalised flux). As expected we find that optical colours correlate with each other and the same holds true for the mid IR/IRAC colours. However mixed optical-mid IR colour-colour diagrams display very weak correlations if any at all. Figure 1 shows an example of such diagrams for NGC 4254, the most actively star forming galaxy in our sample. This galaxy has been chosen because it spans the largest range in colours with its pixels and as such it best illustrates the result. Similar lack of correlation is obtained for all other galaxies. It is worth noting that, although these diagrams indicate that optical colours are independent of mid IR colours for the pixels inside each individual galaxy, the pixels are typically clustered around the barycentre roughly corresponding to the integral colours of the galaxy and do not cover the full parameter space. We find that indeed when all galaxies are put together on these diagrams they form much more correlated sequences, thus lifting the seeming contradiction between the known global colour-colour correlations and the lack thereof that we see on local scales.

The study of local colour-colour correlations within individual galaxies indicates that local SEDs are essentially a two-parameter family: one parameter controls the relative emission of hot dust and PAHs in the mid IR and the other controls the shape of the 


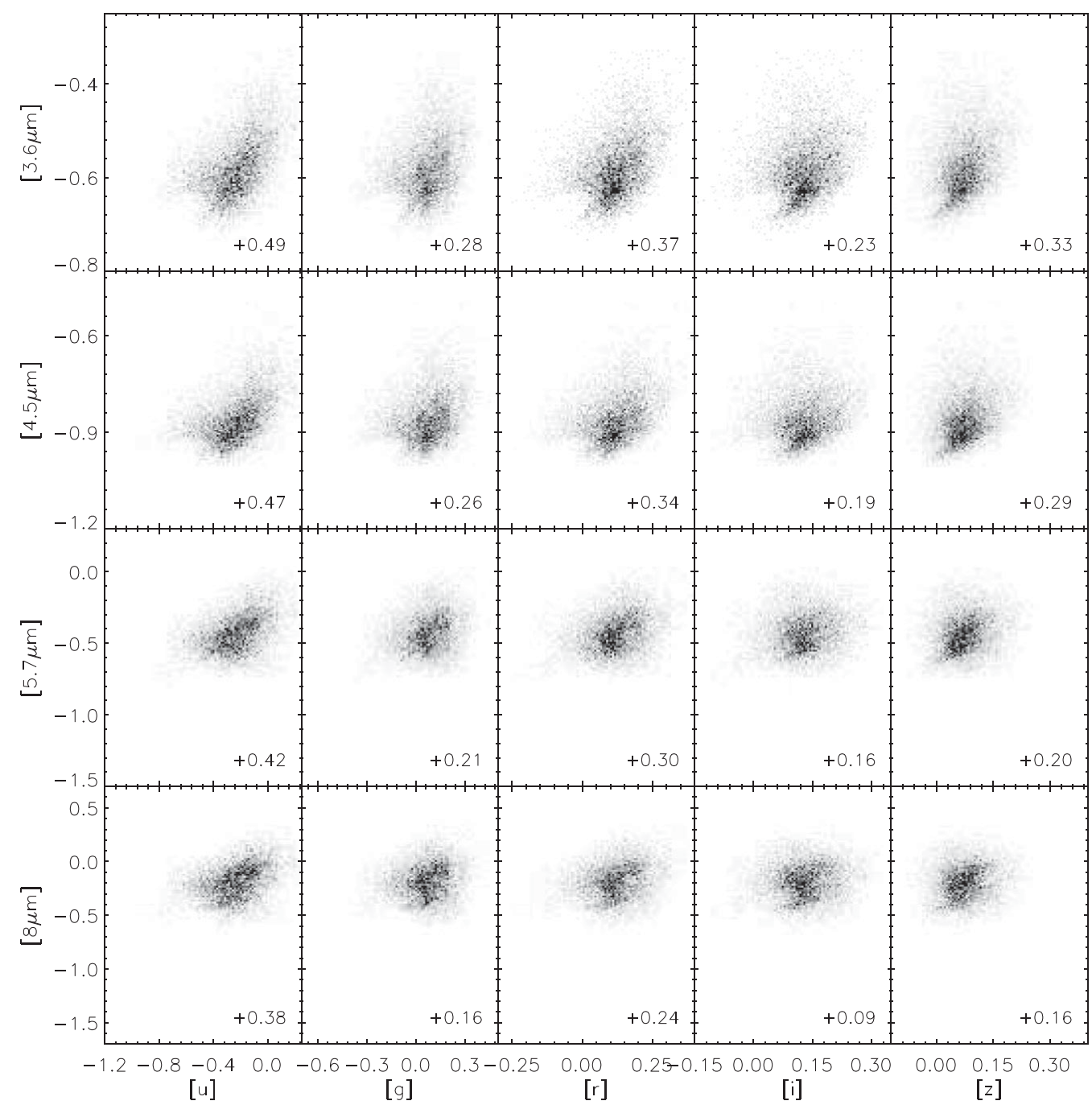

Figure 1. Mid IR-NIR colours vs optical-NIR colours for individual pixels in NGC 4254. The very low degree of correlation between the two spectral regions is also quantified by the low Spearman rank correlation coefficients, in the bottom right corner of each panel. Axis units are $\log \left(\nu_{X} f_{\nu}(X) / \nu_{H} f_{\nu}(H)\right), X$ being the band in the axis title.

optical continuum. We have explored this hypothesis by means of a Principal Component Analysis (PCA) of the pixels SEDs in each galaxy independently. We find indeed that most of the variance is accounted for by the first two principal components (PC), one (PC1) mainly connected to the relative mid IR emission and the other (PC2) to the opical-NIR colours. By mapping the coefficients of PC1 and PC2 we see that they feature distinct galaxy components, such as the bulge, spiral arms, inter-arm regions, young stellar associations and star forming regions. This shows that the optical-IR colour correlations break down already on the scale of this structure and are not just due to stochastic effects nor noise. In order to gain physical insight into the meaning of these PCs, we construct diagrams like those presented in Fig. 2, relative to NGC 4254, for all galaxies. As a function of the surface brightness $(\mathrm{SB})$ in $H$-band $\left(\approx M^{*}\right)$ and in $\mathrm{H} \alpha(\approx \mathrm{SFR})$ we colour code the median coefficient for $\mathrm{PC} 1$ and $\mathrm{PC} 2$ in the left and right panels, respectively. 

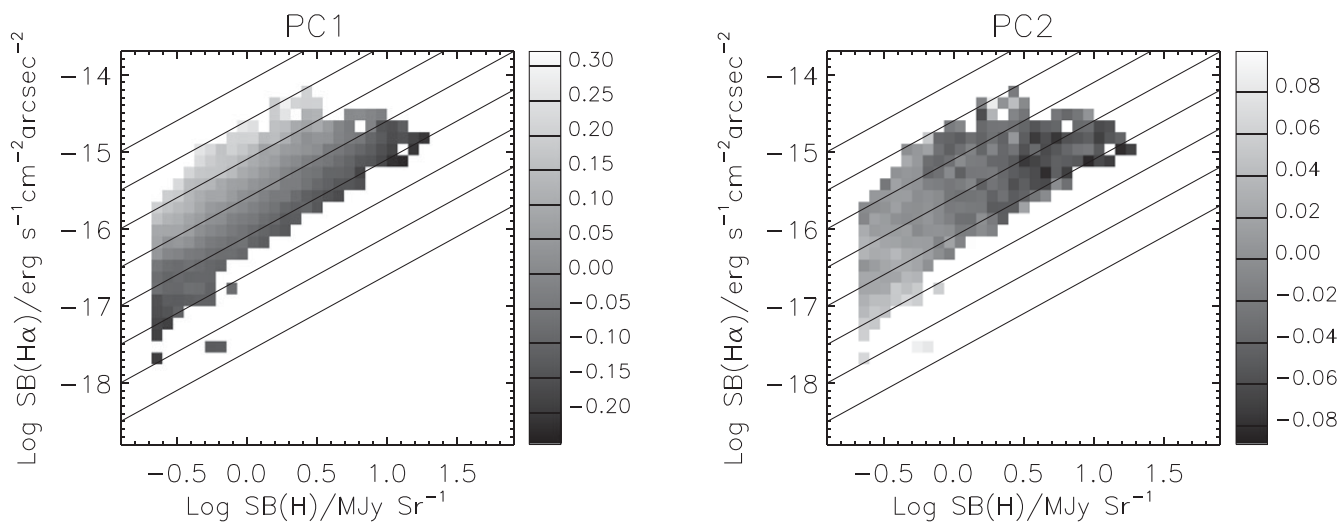

Figure 2. Median coefficients of the two principal components of the local SEDs in NGC 4254 as a function of local surface brightness in $H$-band $\left(\approx M^{*}\right)$ and in $\mathrm{H} \alpha(\approx \mathrm{SFR})$. Diagonal lines are lines of constant $\mathrm{SB}(\mathrm{H} \alpha) / \mathrm{SB}(H) \approx$ specific $\mathrm{SFR}$.

The strength of PC1 (left panel) is tightly correlated to the specific SFR, in that curves of equal PC1 strength are roughly parallel to the lines of constant ratio of $\mathrm{H} \alpha$ over $H$-band intensity. PC2 strength (right panel) is essentially a measure of blue vs red optical-NIR colours and displays only a mild dependence on $\mathrm{SB}(H)$, in line with the well know age gradients in spiral galaxies (e.g. MacArthur et al. 2004) which produce bluer colours in the outer parts of lower SB. This picture is similar for most galaxies with some ongoing star formation, while completely passive galaxies do not have much variation in the mid IR flux. Galaxies like NGC 3521 however show that dust scattering in inclined discs can play a significant role and destroy the PCA properties described so far.

In summary, we find that locally optical-NIR colours are largely uncorrelated from the relative mid IR emission, thus showing that a high surface density of specific star formation does not necessarily imply blue optical colours typical of young stellar populations. In fact, on the one hand a substantial part of the (blue) radiation from young stars can be reddened by dust in correspondence to star forming regions, while on the other hand blue stellar populations may well be present in regions where the star formation has terminated since several hundreds Myr. However, globally these effects average to a relation between current specific SFR and optical-NIR colours. We argue that this is a consequence of the global SF history of a galaxy being relatively smooth on the timescales for such colours to evolve, i.e. $\lesssim 1$ Gyr, such that relatively young stars of age $\gtrsim 100$ Myr are always accompanied by current SF and also the SF episodes extend longer than the times it takes to liberate the young stars from the obscuring birth cloud.

Tight universal mid IR colour correlations. We show that pixels from all galaxies form extremely tight sequences in the mid IR (IRAC) colour-colour plots with typical scatter of a few percent only around the median relations. In practice, at (almost) each pixel of any of our galaxies, given the near IR flux ( $H$ band) and the mid IR flux in one band (e.g. $8 \mu \mathrm{m}$ ) it is possible to predict the flux in the other three IRAC bands to percent accuracy using a universal fitting formula (see Zibetti \& Groves 2011). These relations also highlight the fact that not even at 3.6 or $4.5 \mu \mathrm{m}$ is the stellar emission pure from any dust contaminant. The reason for such a small scatter is currently unclear, although it might be partly related to the small spread in metallicity of our sample. Moreover, by extrapolating these relations to very low mid IR flux relative to $H$, we are able to 
estimate pure stellar colours in the mid IR, which can be extremely useful to separate stellar from dust emission at these wavelengths.

\section{References}

Blanton, M. R., et al. 2003, ApJ, 594, 186

Calzetti, D. et al. 2007, ApJ,666, 870

Gavazzi, G., Boselli, A., Donati, A., Franzetti, P., \& Scodeggio, M. 2003, A\&AA, 400, 451

Hogg, D. W., Tremonti, C. A., Blanton, M. R., Finkbeiner, D. P., Padmanabhan, N., Quintero, A. D., Schlegel, D. J., \& Wherry, N. 2005, ApJ, 624, 162

Kennicutt, R. C., et al. 2003, PASP, 115, 928

Lawrence, A., et al. 2007, MNRAS, 379, 1599

MacArthur, L. A., Courteau, S., Bell, E., \& Holtzman, J. A. 2004, ApJS, 152, 175

York, D. G., et al. 2000, AJ, 120, 1579

Zibetti, S. \& Groves, B. 2011, MNRAS, 417, 812

\section{Discussion}

DA Cunha: Is the very tight correlation you find between the 8 and $5.8 \mu \mathrm{m}$ fluxes an indication of a constant ratio between PAH bands?

ZiBetTi: The relation between the fluxes at 8 and $5.8 \mu \mathrm{m}$ is indeed very tight, with an rms scatter of only a few percent, much less than the $\sim 30 \% \mathrm{PAH}$ variation expected from, for example, the Draine \& Li models. It should be noted that galaxies along the sequence contribute with a very small overlap, such that the small scatter could be a consequence of a roughly homogeneous state inside individual galaxies (especially metallicity). However, all galaxies are very well alligned along the sequence and this supports further the idea of a constant PAH ratio among the galaxies of this small sample. 